http://dx.doi.org/10.12775/szhf.2017.034

RAFA£ Michalski

Nicolaus Copernicus University, Toruń, Poland

E-MAIL: METABASIS1@WP.PL

\title{
Was bedeutet die französische Revolution heute? Die Bemerkungen über Kants Interpretation von signum rememorativum
}

\section{Die wichtigsten gegenwärtigen Interpretationslinien des Erbes der französichen Revolution.}

In diesem Artikel versuche ich Kants ambivalente Einschätzung der Französichen Revolution zu erklären und zu zeigen, dass seine Gedanken zu diesem Thema eine wichtige Bedeutung für unser heutiges Verständnis dieser epochalen „Begebenheit” haben können. Der Sturm auf die Bastille war zweifelsohne ein durbrechendes Ereignis in der Geschichte Europas und für Kant selbst. Wie schon Karl Vorländer schrieb: „[m]an darf nach den uns bisher vorliegenden Quellen, ohne allzugroßer Kühnheit beschuldigt zu werden, die Behauptung wagen, daß ein stärkeres politisches Interesse im Königsberger Philosophen überhaupt erst durch und an einer Revolution, wenn auch nicht der französischen, erwacht ist."' Vorländer überzeugt in demselben Artikel,

${ }^{1}$ K. Vorländer, Kants Stellung zur französischen Revolution, in: Philosophische Abhandlungen. Hermann Cohen zum 70sten Geburtstag dargebracht, Berlin 1912, S. 247. 
dass selbst die Hinrichtung des Königs Ludwig XVI (Januar 1793), „[...] die so manche anfängliche Anhänger abschreckte, den greisen Philosophen in seiner grundsätzlichen Sympathie für die Revolution nicht wankend machen konnte."2 Kants Einstellung zur Französichen Revolution schwankte bekanntich zwischen einer moralischen Verpönung und einer enthusiastischen Anerkennung. Beide Postitionen geraten aber nicht in Widerpsruch zueinander, sondern sie stellen eher zwei verschiedenen Perspektiven seines Philosophierens dar und nämlich: den Standpunkt der Moral und den der Politik. ${ }^{3}$

Diesselbe Ambivalenz scheint auch heute, besonders nach dem Zusammenbruch des Ostblocks (1989) in den öffentlichen Debatten hervorzutreten. $\mathrm{Zu}$ jener Zeit interpretierten manche polnischen Denker die politische Wende der Solidarność-Bewegung im Lichte der französischen Revolution. Oft wiederholte man eine Formel, die Stanisław Brzozowski, der polnische Philosoph (1878-1911), geprägt hat: „Revolutionen sind Augenblicke, in denen die Gesellschaften ihre wesentliche Struktur erkennen." ${ }^{.4}$ In Anknüpfung an diese Formel stellten viele polnischen Intelektuellen fest, dass in achtziger Jahren (zumal nach dem sogenannten Polnischen August 1980) die ofizielle, politische Fassade zerstört wurde und die meisten Bürger die ofizielle gesellschatliche Organisation als rücksichtlosen Mechanismus der Repression begreifen konnten. Vielzitiert war damals Stanisława Przybyszewska, die Autorin des berühmten Dramas Sprawa Dantona, in dem sie Robespierre als politischen Genie darstellte und ihn mit herrschsüchtigem, ehrgeizgem und habgierigem Danton konfrontierte. ${ }^{5}$ Nach Przybyszewska ist jede echte Revolution eine Illumination, die durch die politisch aktivierten Massen erlebt wird. Dadurch überschreiten sie für einen Moment die Grenze der Geschichte, überwinden die Tragik der Situation und die Fatalität der menschlichen Handlungen, ihre Heterotelie. Die Revolutionen haben ihre eigene Logik, eine Logik des Bruchs mit der Geschichte. Sie bringen nämlich die tiefgreifenden Veränderungen nicht nur des politischen Systems, als auch des Alltagslebens im Bereich der Bestimmung von Zeit. Die französische Revolution war darin sehr radikal. Mit dem neuen Kalender wurde bekanntlich der Anspruch der Revolution, eine neue Zeit zu begründen und alle Lebensformen der christlichen Tradition abzustreifen, sichtbar und alltäglich erfahrbar

\footnotetext{
${ }^{2}$ Ibid., S. 269.

${ }^{3}$ Ver. S. Dietzsch, Immanuel Kant. Biografia, tłum. K. Krzemieniowa, Warszawa 2005; M. Żelazny, Idea wolności w filozofii Kanta, Toruń 2001.

${ }^{4}$ S. Brzozowski, Eseje i studia o literaturze, t. 1, Wrocław 1990.

${ }^{5}$ S. Przybyszewska, Sprawa Dantona, Kraków 2003.
} 
gemacht.Trotzdem wurde jedoch die Kontinuität der Geschichte, der Tradition (zumindest teilweise) aufbewahrt. Daraus folgt, dass die revolutionäre Utopie allmählich zu einem Teil der „üblichen” Geschichte wird, obwohl der „utopische” Ausschnitt der Geschichte außergewönlich bleibt, denn die Utopie hat ihm schon ihren unzerstörbaren Stempel aufgedrückt hat. Niemand bestreitet, dass kaum ein Ereignis die Geschichte der Moderne so tief geprägt hat wie die Französische Revolution von 1789 bis 1799. Sie eröffnete eine Phase grundstürzender Transformationen der politischen, sozialen und kulturellen Verhältnisse in Frankreich, aber vor allem hat sie tiefe Spuren in der politischen und sozialen Entwicklung anderer europäischen Länder hinterlassen. Sie wurde zum Motor des Verfassungswandels und der Entstehung liberaler politischer Kulturen, kurzum, sie wurde zum Laboratorium der Moderne, indem sie in der kurzen Spanne eines Jahrzehnts die unterschiedlichsten Verfassungsformen entwickelte, die für die nächsten Jahrhunderte wirkungsmächtig werden sollten. ${ }^{6}$ Zwiefelsohne schuf sie die Grundlagen einer bürgerlich-individualistischen Eigentums- und Gesellschaftsverfassung. Zum ersten Mal entfaltete sie eine demokratische politische Kultur und damit erkämpfte den Durchbruch zur politischen Freiheit. Sie löste einen fundamentalen Prozeß der Politisierung der Gesellschaft und der Ideologisierung der politischen Sprache aus und dabei demonstrierte zugleich die Selbstgefährdung demokratischer Ordnung.

Wie bei Kant dominiert in moderner Literatur zu diesem Thema eine ambivalente Bewertung der Französischen Revolution, die eine simple Alterantive zusammenfasst: sie ist entweder ein durch die Proklamation von Menschen- und Bürgerrechte (1789) symbolisierte Gründungsereignis für eine demokratische politische Kultur oder ein grässliches Mahnmal für uns, dessen Symbol die Hinrichtung des Königs (1793) und „la terreur” - die Schreckensherrschaft ist. Beide Daten werden von Denkern aus verschiedenen politischen Kreisen immer wieder ausgespielt. Sogar für diejenige, die die französische Revolution als ein epochales Ereignis betrachten, ist eine Terrorherrschaft der Jakobiner eine Vorform der totalitären Regime des 20. Jahrhunderts. Sie stimmen damit ein, dass die Revolution die Welt mit der Deklaration der Menschen- und Bürgerrechte sowie der Abschaffung der Feudalgesellschaft, ihrer Privilegien und der Sklaverei zu neuen Ufern des

${ }^{6}$ J. Baszkiewicz, S. Meller, Rewolucja francuska 1789-1794. Społeczeństwo obywatelskie, Warszawa 1983. 
Fortschritts geführt hat, aber zugleich weisen darauf hin, dass sie in ein Meer von Blut mündete.

Das ganze 19. und 20. Jahrhundert über wurde über Fluch und Segen des Jahres 1789 und 1793 gestritten. Die Marxisten und radikalen Linken idealisierten dieses Ereignis, die Konservativen verwarfen im Allgemeinen das revolutionäre Erbe, die Liberalen nahmen dagegen am meisten eine ambivalente Haltung demgegenüber ein, obwohl manche von ihnen meinten, dass der Zusammenbruch des Kommunismus ein Ende der Epoche bedeutet, die in 1789 begann und zu katastrophalen Folgen führte. Zum Beispiel Francois Furet hält die französische Revolution für eine Matrix des Totalitarismus und deswegen schlägt vor, sie als eine geschichtliche und politische Anomalie und nicht als das Gründungsereignis der liberalen Demokratie zu betrachten. ${ }^{7}$ Nach diesem französischen Historiker zeigt das Beispiel des Englands, dass die Grundsätze der persönlichen Freiheit, der Gleichheit, etc. auf gewaltlose, vernünftige Weise erreicht werden konnten. In diesem Gesichtspunkt trifft er sich mit dem Standpunkt des Konservatismus - die französische Revolution sei ein Gründungsereignis der totalitären Regime und eine Wurzel der schlimmsten Todsünden des zwanzigsten Jahrhunderts: Holocausts, Gulags und der Terroranschläge am 11. September 2001.

Nach der mehr niuancierten Ansicht waren die meisten Revolutionäre persönlich ehrlich und sogar tugendhaft, aber aus verschiedenen Gründen wurden sie zu Opfern des Fanatismus, der sich als höchst gefährlich und folgenschwer herausstellte. Daraus folgt jedoch eine zynische Klugheit: die Korruption ist besser als ethische Reinheit, eine Machtgier als eine Besessenheit durch seine Mission. Sophie Wahnich in ihrem durchbrechenden Werk In defence of terror. Liberty or death in French Revolution ${ }^{8}$ polemisiert mit dieser vorherrschenden doxa zum Thema der französischen Revolution. Aufgrund der akribischen Analysen der nacheinander folgenden Etappen des Terrors zeigt sie, dass der politische Umbruch der Jakobiner kein Ausbruch der hemmungslosen Gewalt war, sondern ein präzis geplanter und in reichem Maße kontrollierter Versuch, eine solche Explosion vorzubeugen. Ähnlich wie Hannah Arendt stellt sie die These auf, sowohl liberale Demokraten, als auch Marxisten haben das Drama der neuzeitlichen Revolutionen nicht verstanden, weil sie beide nicht begriffen haben, dass das eigentlich Revolutionäre

\footnotetext{
${ }^{7}$ F. Furet, Interpreting the French Revolution, Cambridge 1978, p. 12.

${ }^{8}$ S. Wahnich, In defence of terror. Liberty or death in French Revolution, tr. D. Fernbach, London 2012.
} 
in den modernen Revolutionen immer wieder ein mißlungener Versuch der Constitutio Libertatis war - ein Versuch der Errichtung eines politischen Raumes öffentlicher Freiheit, in dem die Menschen als gleiche und freie Bürger ihre gemeinsamen Angelegenheiten in die eigene Hand nehmen. ${ }^{9}$ Beide, die Liberalen und Marxisten, waren gleichermaßen einem Verständnis verhaftet, welchem nach der Endzweck der Politik etwa jenseits der Politik sei - sei es die Verfolgung der individuellen Interessen, die private Wohlfahrt der Bürger oder die Etablierung einer klassenlosen Gesellschaft. Arendts Behauptung, das die französische Revolution scheiterte, weil sie sich auf die sogenannte soziale Frage, auf die Befreiung vom Lebensnot und versuchte die Leidenschaft de Mitleidens in den Bereich der Politik einzuführen. Daraus folgt, Arendt nach, eine Tugend-Terror der Robespierre-Diktatur: „[...] wo immer man Tugend aus dem Mitleid abgeleitet hat, haben sich Grausamkeiten ergeben, die es unschwer mit den grausamsten Gewaltherrschaften der Geschichte aufnehmen können. " ${ }^{10}$ Wahnich beweist, dass eine solche Deutung unbegründet ist. Die revolutionäre Rituale, Gesetzgebung und verschiedene Formen der militärischen, administrativen und polizeilichen Selbstorganisation dienten der Hemmung der massenhaften, unkontrollierten Aggression. Die Revolutionäre setzten sich zum Ziel, die Zirkulation der Emotionen durch einen neuen juridischen Diskurs zu steuern. ${ }^{11}$ Es ging ihnen nicht um die Befreiung von der Not, sondern um die Wiederherstellung der Würde jeder Bürger, d.h. darum, allen allgemeine Rechte und die Möglichkeit der politischen Teilnahme zu gewähren. ${ }^{12}$

Im Zusammenhang mit Kants Interpretation der französischen Revolution ist wichtig, dass er dieses epochale Ereignis als das Zeichen des ankommenden Fortschritts der menschlichen Gattung, als Funke der politischen Freiheit verstand hat. Er konnte natürlich Wahnichs Auffasung nicht teilen, weil er vehemment jeden Aufstand gegen den Souverän ablehnte. Der gewaltsame Widerstand widerspricht unabhängig von seinen Intentionen dem Recht selbst und dem gesunden Menschenverstand. Trotzdem drückt Kant einen Lob auf die Revolution aus. In Streit der Fakultäten schreibt er: „[...] ein solches Phänomen in der Menschengeschichte vergißt sich nicht mehr, weil es eine Anlage und ein Vermögen in der menschlichen Natur zum Bes-

\footnotetext{
${ }^{9}$ H. Arendt, Über die Revolution, München 1974, S. 85, 160.

${ }^{10} \mathrm{~S}$. Wahnich, In defence of terror..., op. cit., p. 114.

${ }^{11}$ Ibid., p. 19.

12 Ibid., p. 85.
} 
seren aufgedeckt hat, dergleichen kein Politiker aus dem bisherigen Laufe der Dinge herausgeklügelt hätte [...]”. Ja wenn selbst, „[...] die Revolution oder Reform der Verfassung eines Volks gegen das Ende doch fehlschlüge, [...]" so ist doch „[...] jene Begebenheit zu groß, zu sehr mit dem Interesse der Menschheit verwebt und [...] zu ausgebreitet, als daß sie nicht den Völkern bei irgendeiner Veranlassung günstiger Umstände in Erinnerung gebracht und zur Wiederholung neuer Versuche dieser Art erweckt werden sollte." ${ }^{13}$ Wir können also sagen, dass Kant in diesem Abschnitt, der "Wahrsagende Geschichte der Menschheit" überschrieben ist, die späteren Revolutionen, von der französischen 1830 bis zur russischen Oktoberrevolution, im gewissen Sinne vorausgesagt. Wir müssen uns doch dessen bewußt sein, dass Kants Affirmation beschränkt war. Er schätzt zwar die revolutionären Ereignise hoch, weil sie deutlich auf die Idee der Freiheit und den Fortschritt der Menshlichkeit verwiesen, aber zugleich postuliert er einen reformistischen Weg für die zukünftigen Veränderungen. Diese Begebenheit, schreibt er in Streit der Fakultäten, die das ganze Menschengeschlecht angeht, diese „[...] allgemeine und uneigennützige Teilnahme”, mit der man dem „erhofften Gelingen” des großen Unternehmens „zujauchzt”, ist ihrerseits das Phänomen nicht einer Revolution, sondern „[...] der Evolution einer naturrechtlichen Verfassung, die zwar nur unter wilden Kämpfen noch nicht selbst errungen wird [...], die aber doch dahin führt, zu einer Verfassung hinzustreben, welche nicht kriegssüchtig sein kann, nämlich der republikanischen." ${ }^{14}$

In nächsten Abschnitten des Artikels versuche ich Kants Ansichten zu diesem Thema skizzieren, um am Ende zu zeigen, dass seine Deutung der Revolution eine interessante, obgleich peinliche für uns Perspektive eröffnet, mit dem ambivalenten Erbe der französischen Revolution zurrechtzukommen.

\section{Die Revolution und die Politik}

Am Anfang muss man sagen, dass Kant keine systematische Theorie der Politik und des politischen Handelns herausgearbeitet hat. Solche Schriften wie Metaphysik der Moral oder Zum ewigen Frieden zeigen eindeutig, dass die Politik sich nur im Rahmen ereignen kann, der durch Verfassung und

\footnotetext{
${ }^{13}$ I. Kant, Der Streit der Fakultäten, AA, 07: 88.

${ }^{14}$ Ibid.
} 
Rechtsordnung gebildet wird. Die Politik im Gegegensatz zur Moral dient dem allgemeinen Wohlstand und soll das friedliche Zusammenleben der Staaten und Einzelnen sichern. Im Streit der Fakultäten legt aber Kant nahe, dass eine echte Politik den Raum einer zwischenmenschlichen Freiheit eröffnet und die Französische Revolution als ein paradigmatischer Fall des politischen Handelns verstanden werden kann. Die Revolution zerstört zwar die alte soziale und politische Ordnung, aber zugleich befreit sie die Revolutionäre aus Fesseln ihres privaten Lebens, aus ihrer egoistischen Interessen und weist ihnen die universelle Idee der Freihet. Vor allem initiiert sie ein neues, einmaliges Ereignis im Bereich der menschlichen Angelegenheiten, dessen Resultat tiefgreifende politische Systemveränderungen sind. Die Revolution ist von Natur aus irrational, aber sie erzeugt die politische Öffentlichkeit - die sogennante Publizität, die diesem Ereignis einen Sinn gibt, der über die partikularen Motivationen und Vorstellunegn der Revolutionäre selbst hinauskommt. Mit anderen Worten, die Revolution ohne enthusiastich reagierendes und diskutierendes Publikum würde nicht die Schwelle des Politischen überschreiten und bliebe nur einen Ausdruck der blossen Gewalt. Erst in Verbindung mit dem urteilenden Publikum wird die Revolution ein politisches Ereignis par excellence.

\section{Die rechtlich-moralische Bewertung der Revolution}

In Streit der Fakultäten bewertet Kant die Französische Revolution positiv und betrachtet sie als ein wichtiges, historisches Ereignis, obgleich er in seinen anderen Werken ein Widerstandsrecht des Volkes und des Einzelnen auch gegen evident ungerechte Gesetze - eindeutig ablehnt. Kant begründet seine Ablehnung des Widerstandsrechts mit Hilfe von drei Argumenten. Ich referiere sie sehr kurz:

1. Das erste - nennen wir es - Glückseligkeitsargument bezieht sich auf die Situation, in der ein Volk die herrschende Machtordnung abschaffen will, um seinen Wohlstand zu verbessern. Die Revolutionäre streben an die Veränderung der Staatsform, weil das bestehende System dem Gemeinwohl nicht (mehr) dient. Nach Kant ist solche Motivation nicht nur trügerisch, sondern vor allem destruktiv für die Rechtsordnung. Sie ist trügerisch, weil unsere Vorstellungen von dem glücklichen, gelungenen Leben sich im Zeitablauf ändern und von Natur aus unbestimmt sind. Sie ist destruktiv, weil sich die 
Rechtsordnung auf das allgemeine Vernunftrecht und nicht auf launischen Neigunen stützen soll. Kurzgesagt, die Revolutionäre wollen eigene Glückseligkeit auf Kosten der obersten Maximen eines Staates erreichen, was verdammenswert ist.

2. Von zentraler Bedeutung ist für Kant das logische Argument. Es geht in aller Kurze so: Indem jeder, der im Namen eines Widerstandsrechts auftrete, sich die Stellung des Souveräns anmaße, entstehe ein logischer Widerspruch zum wirklichen Souverän. „Denn um zu demselben [dem Widerstand] befugt zu sein, müßte ein öffentliches Gesetz vorhanden sein, welches den Widerstand des Volkes erlaubte, d. h. die oberste Gesetzgebung enthielte eine Bestimmung in sich, nicht die oberste zu sein [...]; welches sich widerspricht."15

Das Volk soll also kein Recht des Aufstands haben, weil es dem Recht selbst und dem gesunden Menschenverstand widerspricht. Die Revolution setzt eine tödliche Gewalt frei und zerstört die Verfassung, die eine notwendige Grundlage für den Gesellschaftsvertrag bildet. Wenn die Verfassung ein Recht des Aufstandes zulässe, wäre ein zusätzliches, oberes Verfassungsorgan notwendig, aber das widerspricht der Idee der Verfassung selbst.

4. Nach dem dritten Argument bedeutet jeder Widerstand, jede Revolution ganz einfach einen Schritt zurück in Richtung des Naturzustandes.

Aus den oben skizzierten Argumenten folgt Kants sehr kritische Beurteilung jedes Aufstandes gegen den Staat. In Metaphysik der Sitten schreibt er folgendes: „Wider das gesetzgebende Oberhaupt des Staats giebt also keinen rechtmäßigen Widerstand des Volks; denn nur durch Unterwerfung unter seinen allgemein=gesetzgebenden Willen ist ein rechtlicher Zustand möglich; also kein Recht des Aufstandes (seditio), noch weniger des Aufruhrs (rebellio), am allerwenigsten gegen ihn als einzelne Person (Monarch) unter dem Vorwande des Mißbrauchs seiner Gewalt (tyrannis) Vergreifung an seiner Person, ja an seinem Leben (monarchomachismus sub specie tyrannicidii). Der geringste Versuch hiezu ist Hochverrath (proditio eminens), und der Verräther dieser Art kann als einer, der sein Vaterland umzubringen versucht (parricida), nicht minder als mit dem Tode bestraft werden." 16

Kant lehnt damit jeden Aufstand, also jede Revolution gegen den Staat, aber lässt die allmähligen, politischen Veränderungen auf dem Weg der Reformen zu. Entscheidend ist hier das Prinzip der Publizität, die die Überein-

\footnotetext{
${ }^{15}$ I. Kant, Die Metaphysik der Sitten, AA, 06: 322.

${ }^{16}$ Ibid., S. 320.
} 
stimmung der politischen Reformen mit der Rechtsordnung und der Moral verbürgen soll.

\section{Die Publizität als Methode der Aufklärung}

Kant begreift die Publizität als Methode der Aufklärung, also der Befreiung des Menschen aus selbstverschuldeter Unmündigkeit. Im Hinblick auf den Einzelnen bezeichnet diese eine subjektive Maxime, nämlich selbständig und kritisch zu denken. Im Hinblick auf die Menschheit im ganzen bezeichnet sie ein Streben nach der vollkommen gerechten Ordnung. In beiden Fällen muß Aufklärung durch die Publiztät vermittelt werden: „Es ist für jeden einzelnen Menschen schwer, sich aus der ihm beinahe zur Natur gewordenen Unmündigkeit herauszuarbeiten. [...] Daß aber ein Publikum sich selbst aufkläre, ist eher möglich; ja es ist, wenn man ihm nur Freiheit läßt, beinahe unausbleiblich." ${ }^{17}$ Aus der Perspektive der Aufklärung fällt das kritische Selbstdenken, also der Gebrauch der Vernunft mit dem öffentlichen Gebrauch der Vernunft zusammen. In erster Linie ist dies eine Aufgabe der Gelehrten,besonders derjenigen, die mit Prinzipien reiner Vernunft zu tun haben, also der Philosophen. Kant beschreibt der Streit der Fakultäten als die kritische Auseinandersetzung der unteren mit den oberen Fakultäten. Die oberen, nämlich, Theologie, Jurisprudenz und Medizin, gründen in der einen oder anderen Weise auf Autorität. Sie unterstehen auch der staatlichen Aufsicht.

Dagegen haben die unteren Fakultäten mit Erkenntnissen aus reiner Vernunft zu tun. Deren Vertreter, die Philosophen, lassen sich, vom Interesse der Regierung unabhängig, allein von der Vernunft leiten. Ihr Geist „[...] geht auf öffentliche Darstellung der Wahrheit"18. Die Vernunft muß in solchem Streit der Fakultäten „[...] öffentlich sprechen berechtigt sein muß, weil ohne eine solche Wahrheit (zum Schaden der Regierung) nicht an den Tag kommen würde". ${ }^{19}$ Und zwar, wie Kant hinzufügt, zum Schaden der Regierung selbst. Die Öffentlichkeit, innerhalb derer die Philosophen ihre kritische Reflexion betreiben, ist jedoch keine bloß akademische Öffentlichkeit. Im öffentlichen

\footnotetext{
${ }^{17}$ I. Kant, Beantwortung der Frage: Was ist Aufklärung?, AA, 8:33.

${ }^{18}$ I. Kant, Der Streit der Fakultäten, AA, 07:33.

${ }^{19}$ Ibid., S. 20.
} 
Gebrauch der Vernunft können nämlich alle, die sich darauf verstehen, teilnehmen. Sie müssen aus den Schranken ihrer Privatsphäre hervortreten und sich an ein räsonierendes Publikum, an „eine Leserwelt” durch Schriften wenden. Dieses Publikum bilden vor allem Literaten, der Salons, in denen sich „die gemischten Gesellschaften” diskutierend austauschen.

Die revolutionäre Politisierung der intelektuellen Eliten entsprach im beträchtlichen Maße den Forderungen, die Kant in Streit der Fakultäten an die aufklärische Öffentlichkeit gestellt hat. Diese politische Öffentlichkeit bedeutete die Mitgliedschaft in einem politischen Klub oder in einer Volksgesellschaft (sociétés populaires). Das organisatorische Spektrum der Klubs reichte von intellektuellen Diskussionszirkeln über politisch-parlamentarische Vereinigungen mit geregelten Mitgliedsbeiträgen bis hin zu den wesentlich offeneren Volksgesellschaften. Alle diesen Vereinigungen während der französischen Revolution dienten der politischen Information, Diskussion, Beobachtung des politischen Geschehens und dem Versuch seiner Beeinflussung. Die Vereinigungen waren zum Teil intellektuelle Diskussionszirkel, zum Teil Gruppen mit geregelten Mitgliedsbeiträgen wie der Jakobinerklub (Club des Jacobins). ${ }^{20}$ Die zahlreichen politischen Clubs und Volksgesellschaften, die 1788/89 ins Leben gerufen wurden, bauten auf die seit der Aufklärung etablierten bürgerlichen Organisationsformen der Salons, Lesezirkel, akademischen Gesellschaften und Freimaurerlogen auf, öffneten sich aber einem größeren Kreis und neuen Zielen. Sie verstanden sich als Orte des Austauschs und der gemeinsamen politischen Willensbildung und konstituierten sich teilweise bewusst als parallele Einrichtungen zu den Sektionen und anderen politischen Organen, um die Arbeit von Verwaltung und Regierung zu kontrollieren und die neuen staatsbürgerlichen Rechte aktiv beobachten. Eine eminente Rolle in der Verbreitung neuer Ideen spielte dabei die Presse. im Unterschied zum Ancien Regime erschienen die Zeitungen häufiger, also in der Regel wöchentlich und nicht mehr monatlich. Sie waren darum aktueller und steigerten ihre Auflage während der Revolutionszeit.

Kant musste von dieser aktiven Publizität begeistert werden. Die Öffentlichkeit der Philosophen, Literaten, Publizisten, usw. soll, seiner Meinung nach, nicht nur einer kollektiven Aufklärung dienen, nicht nur ein Veto gegen Mißbräuche der Regierenden ankündigen und Pathologien des Rechtssystems verurteilen, aber auch zwischen der Stimme des Volkes und der regierenden Macht vermitteln. Seine positive Beurteilung der Revolution er-

${ }^{20}$ J. Baszkiewicz, S. Meller, Rewolucja francuska..., op.cit., s. 88-127. 
folgte daraus, dass er selbst eine Perspektive des unbeteilgten, distanzierten und räsonierenden Publikums einnahm und dadurch wahrscheinlich kein Widerspruch mit seiner moralischen Verpönung empfinden musste.

\section{Der Entusiasmus und das enthusiastische Urteil}

Kant war freilich sich im Klaren darüber, dass die wichtigsten Triebfedern der Französischen Revolution soziale Gefühle des Unrechts, Leidens und der Unzufriedenheit waren. Das unterdrückte Volk hatte weder Geduld, noch Vertauen zum räsonierenden Publikum, deswegen handelten Revolutionäre unter dem entscheidenden Einfluss der explosiven Emotionen, aber zugleich - was Kant immer wieder betont - haben sie in der Menscheit eine Idee der Freiheit gewiesen und die Hoffnung auf den Fortschritt ganzer Gattung geweckt. Weil die Revolution auf brutale Weise die herschende Rechtsordnung und die Verfassung zunichte gemacht hat, konnte sie eine morale Zustimmung bei Kant nicht finden. Es gibt für ihn keine Möglichkeit, ihre Grausamketen zu legitimieren, als heilige der Zweck die Mittel. Aus moralischer Perspektive ist sie eindeutig falsch und verdammenswert.

Kants Enthusiasmus bezog sich nicht auf konkrete Ereignisse, Handlungen, die sich in der Revolution abgespielt haben, sondern auf einen tieferen politischen Sinn, den erst das aufgeklärte Publikum aus der raumzeitlichen Distanz wahrnehmen und dann zur öffentlichen Debatte stellen konnte. Kant schließt nicht aus, dass aufgrund dieser Debatte (auch mit Regierenden) Postulate aufgestellt werden können, die zur Verbesseung des politischen Systems in Zukunft beitragen.

In Streit der Fakultäten stellt Kant die Frage, ob sich in der Französichen Revolution für den Menschen ein „Fortschreiten seiner Gattung zum Besseren" erkennen lasse. Als Maßstab dient für ihn eine enthusiastische Reaktion der Zuschauer, also derjenigen, die gar nicht direkt an der Revoltion beteiligt waren. Eben diese Reaktion lässt die Revolution als "Geschichtszeichen” (signum rememorativum) ansehen. Der Enthusiasmus der Zuschauer ist natürlich nicht deswegen ausschlaggebend für die politische Bedeutung der Revolution, weil durch ihn konkrete Taten verübt wurden. Seine Rolle besteht auch nich darin, die Zuschauer selbst zu aktivieren, denn der Enthusiasmus hängt weder vom Gelingen, noch vom Scheitern, oder überhaupt von den realen Möglichkeiten, eine solche Revolution durchführen zu können, ab. Die 
enthusiatische Reaktion ist so wichtig für Kant vor allem aus diesem Grunde, dass erst und nur sie eine politische Handlung als solche konstituiert. Unabhängig von dieser Reaktion ist das politische Ereignis im eigentlichen Sinne nicht vorhanden, sondern zerfällt in eine chaotische Kette von Taten. Die politische Handlung ohne das Urteil über sie ist nur ein blindes Treiben von Individuen oder Kollektiven. ${ }^{21}$

Kant beschreibt die Revolution als eine Art von Drama oder Theateraufführung. Demnach gibt es die Handelnde, Akteure, oder Spielende, andererseits die Zuschauer und natürlich eine geschichtliche Szene. Dabei müssen Akteure gar nicht wissen, was sie tun. In meisten Fällen sind sie nicht imstande, einen ideellen Sinn zu ergreifen, den sie durch ihre Taten bilden. Die von Kant benutzte Theatermetaphorik verweist auch darauf, dass der $\mathrm{Zu}$ schauer einen angemessenen Platz einnehmen muss, der ihm erlaubt, sich über die Zufälligkeit der Tatsachen zu erheben und das „Ganze” zu erblicken. Der Akteur ist dagegen nur ein Teil des Spektakels und muss seine Rolle wohl oder übel spielen, deswegen ist er immer parteiisch. Im Gegenteil nimmt der Zuschauer eine unparteiische Haltung des unbeteilgten Beobachters ein - er hat keine ihm von oben zugeschriebene Rolle in dem geschichtlichen Drama. Diese distanzierte Haltung bildet nach Kant eine notwendige Bedingung der Urteilskraft, die das revolutionäre Ereignis beurteilt.

Das Urteil des Publikums darf man weder mit entusiastischer Reaktion der Menschenmenge z.B. angesichts eines Silvesterfeuerwerks, welcher schnell

${ }^{21}$ Kant schreibt: „Diese Begebenheit besteht nicht etwa in wichtigen, von Menschen verrichteten Thaten oder Unthaten [...]Nein: nichts von allem dem. Es ist bloß die Denkungsart der Zuschauer, welche sich bei diesem Spiele großer Umwandlungen öffentlich verräth und eine so allgemeine und doch uneigennützige Theilnehmung der Spielenden auf einer Seite gegen die auf der andern, selbst mit Gefahr, diese Parteilichkeit könne ihnen sehr nachtheilig werden, dennoch laut werden läßt, so aber (der Allgemeinheit wegen) einen Charakter des Menschengeschlechts im Ganzen und zugleich (der Uneigennützigkeit wegen) einen moralischen Charakter desselben wenigstens in der Anlage beweiset, der das Fortschreiten zum Besseren nicht allein hoffen läßt, sondern selbst schon ein solches ist, so weit das Vermögen desselben für jetzt zureicht. haben vor sich gehen sehen, mag gelingen oder scheitern; sie mag mit Elend und Greuelthaten dermaßen angefüllt sein, daß ein wohldenkender Mensch sie, wenn er sie zum zweitenmale unternehmend glücklich auszuführen hoffen könnte, doch das Experiment auf solche Kosten zu machen nie beschließen würde, -diese Revolution, sage ich, findet doch in den Gemüthern aller Zuschauer (die nicht selbst in diesem Spiele mit verwickelt sind) eine Theilnehmung dem Wunsche nach, die nahe an Enthusiasm grenzt, und deren Äußerung selbst mit Gefahr verbunden war, die also keine andere als eine moralische Anlage im Menschengeschlecht zur Ursache haben kann." (I. Kant, Der Streit der Fakultäten, AA, 07: 85). 
und oberflächlich entflammt und ebenso schnell verpufft, noch mit fanatischer Euphorie und Begeisterung der politisch agitierten Massen identifizieren. Der vernünftige Enthusiasmus des aufgeklärten Publikums führt nicht zur Entpolitisierung oder zur Ästhetisierung der Politik, ganz im Gegenteil bildet er einen Raum der politischen Freiheit. Es geht hier also nicht um eine passive Akklamation, um physiologische Anregung, sondern um einen geistigen Zustand - d.h. der Enthusiasmus hat einen tieferen Sinn nur dann, wenn er sich mit dem Urteil der Zuschauer verbindet. Das enthusiastische Urteil ist dabei strukturanalog zum ästhetischen Urteil.

\section{Das enthusiastische Urteil als Modus der ästhetischen Urteilskraft}

Um die genaue Struktur dieses Urteils zu bestimmen, muss es zunächst gegen die bestimmende Urteil abgegrenzt werden. Das Revolutionäre jeder Revolution beruht darauf, dass sie auf etwas Neues, Künftiges, Ungedachtes verweist. Das bestimmende Urteil würde dieses „unbestimmte Neue” völlig verfehlen, denn es subsummiert - laut Definition - das Besondere unter das Allgemeine; es würde damit die revolutionäre Situation anhand schon vorhandener Begriffe beurteilen. Ein Urteil über revolutionäre Taten kann sich also nicht aus einer bestehenden Praxis herleiten, da in einer Revolution die Begriffe und die fundierenden sozialen Praktiken, anhand derer die Erscheinungen subsumiert werden könnten, noch nicht zur Verfügung stehen.

Die historische Einordnug der Französichen Revolution kann erst in der Zukunft erfolgen. Obwohl der Enthusiasmus also kein bestimmendes Urteil sein darf, darf er auch nicht inhaltsleer sein. Denn dann wäre, was in der Revolution stattfindet, ganz beliebig und nur Anlass für ein abstraktes, enthusiastisches Urteil. Diese beiden Optionen verwirft Kant, wenn er davon spricht, der Etnthusiasmus richte sich nicht auf konkrete Ereignisse, sondern auf eine Idee.

In der Französichen Revolution kommt es zum radikalen Bruch mit dem Vergangenen und zu einer Neugründung des Politischen, welche nicht durch eine Kette von kausalen Verbindungen mit dem Alten verknüpft ist und nicht dessen Fortsetzung darstellt. Die politische Freiheit besteht also für Kant darin, dass die Revolutionäre selbst „Urheber” neuer Ketten der Ereignisse sind. 
Wenn die Revolution Neues in die Welt bringt, dann geschieht in der revolutionären Handlung immer etwas prinzipiell Unbestimmbares, etwas, was sich durch alte Konzeptionen nicht beschreiben lässt. Die Revolutionäre verfügen über keine fertigen, überprüften Maximen der Handlung, über keine Muster für die Entscheidungsfindung, deswegen handeln sie wie Kant schreibt - gleichsam „ins Leere” hinein. Doch der Bruch mit dem überkommenen Regime, welche die Französische Revolution darstellte, zeigt zunächst, dass es möglich ist, aus dem Tradierten auszubrechen. Aber nicht jeder Neuanfang setzt sich die Gründung einer freiheitlichen Ordnung zum Ziel - nicht jede Revolution ist demokratisch. Deswegem muss das politische Urteil auch die Antwort darauf beinhalten, ob es sich um eine freiheitliche Revolution handelt - nur dann markiert die Französische Revolution, in Kants Worten, ein „Geschichtszeichen”.

Der echt politische Enthusiasmus wird nach Kant nur ausgelöst, wenn bei den revolutionären Geschehnissen Ideen der Vernunft im Spiel sind. Das Geschichtszeichen richtet sich ,aufs Idealische” und kann „keine andere, als moralische Anlage im Menschengeschlecht zur Ursache haben". Der Ausdruck „moralische Anlage” bezieht sich in diesem Zusammenhang natürlich auf die Idee der Freiheit. Der Enthusiasmus muss also über das formale Kriterium des Bruchs mit der Vergangenheit hinaus auch noch das normative Urteil enthalten, ob dieser Bruch freiheitlichen Ideale folgte. Aber auch dafür findet sich kein Kriterium in den Taten der Revolution selbst.

Wie kann man nun ein Urteil beschreiben, welches stattdessen nur durch die Idee der Freiheit hervorgerufen wird? Die Freiheit als Idee ist doch kein Objekt, es ist nicht möglich, diese Idee direkt zu verwirklichen. Kant verweist in diesem Kontext darauf, dass der Enthusiasmus gerade deswegen adäquat ist, eine solche Situation zu beurteilen, weil er in seiner Struktur objektlos ist, sich also gar nicht auf die konkreten Geschehnisse bezieht.

Wie diese objektlose Struktur des Enthusiasmus zu verstehen ist, erhellt ein Blick auf Kants Diskussion des Enthusiasmus in der „Analytik des Ehabenen" der Kritk der Urteilskraft. Der Enthusiasmus ist analog zum dynamischen Erhabenen, da er ebenfalls durch Ideen ausglöst wird. Im objektlosem Erhabenen ist der innere, affektbesetzte Zustand der Vermögen ein „Gegenstand” des Gefühls. Ähnlich wie das Erhabene ist also der Enthusiamus kein intentionales Gefühl. Beide Gefühle sind zwar durch formlose, unbegrenzte Erscheinungen ausgelöst, aber letztendlich beziehen sie sich auf eine unvorstellbare Idee der Vernunft. Im Erhabenen und im Enthusiasmus kommt ein Urteil zum Ausdruck in der Form des Affekts, welcher in der lustvol- 
len Reaktion auf die Erfahrung der Zweckmäßisgkeit seiner Vermögen in der negativen (indirekten) Darstellung einer Idee reagiert. Ideen sind wie im Gefühl des Ehabenen nur „negativ” darzustellen: das Individuum erlebt es paradoxerwiese als lustvoll, dass sein Vernunftvermögen scheitert. Das Erhabene ist gekennzeichnet durch das Gefühl des Überwältigtwerdens, bei dem wir mit etwas konfrontiert werden, das für unser Vorstellungsvermögen inadäquat ist, also mit etwas, was wir nicht darstellen können. Das, was unsere Einbildungskraft zwingt, etwas zu tun, was ihr Vermögen übersteigt, liegt nicht in den Sinneseindrücken selbst, sondern in der Vernunft, die uns zwingt, das Unvorstellbare zu vorstellen. Das Scheitern der Einbildungskraft erzeugt Unlust, aber der negative Verweis auf das Vernunftvermögen erzeugt Lust.

Der ähnliche Prozess geht beim Gefühl des Enthusiasmus vor. Das Publikum der Revolution wird mit dem Chaos politischer Ereignisse, mit ihren Grausamkeiten usw. konfrontiert, was bei ihm Unlust (das Entsetzen, Schauder, Angst usw.) erzeugt, aber zugleich - Lustgefühl, d.h. eben den Enthusiasmus, der sich auf die Idee der Freiheit bezieht.

Der Enthusiasmus ist jedoch nicht nur aufgrund seiner Objektlosigkeit und seines Bezugs zu den Ideen der Vernunft ein Modell für politisches Urteilen, sondern gerade aufgrund seiner affektiven Struktur: denn als Affekt ist er nicht das Andere der Vernunft, was für ein politisches Urteil problematisch wäre. In diesem Gefühl zeigt sich die Vernunft selbst, und nämlich in Form des Urteilens. Das heißt, der Enthusiasmus als von Unlust und Lust strukturierter Affekt is die Form, in der sich die Vernunft im Moment der Revolution äußert und zugleich „zeigt”, dass die Kategorien, anhand derer das Neue zu beurteilen ist, noch nicht zur Verfügung stehen, die Handlungen aber trotzdem nich arbiträr und chaotisch sind, sondern sie als Ausdruck der Freiheit angesehen werden können.

Die Parallelisierung mit dem Erhabenen enthüllt noch eine Konvergenz beider Gefühle. Nach Kant ist kein Gegenstand der Natur selbst als „erhaben" zu beurteilen, sondern bestenfalls als geeignet, um die Erhabenheit im beurteilenden Subjekt selbst darzustellen. Ähnlich der Enthusiasmus der Zuschauer handelt gar nicht von der Französischen Revolution - sondern von den Zuschauern. Ob eine Handlung freie Handlung war, kann also nur im affizierten Urteil der Zuschauer bestimmt werden.

Das enthusiastische Urteil als Modus der Urteilskraft gibt keine Sicherheit, aber es erhebt den Anspruch auf Universalität. Seine Glaubwürdigkeit hängt von der öffentlichen Debatte, von dem richtigen Gebrauch der Vernunft al- 
ler, die an dieser Diskussion teilnehmen. Die Politik, die mit der Zufälligkeit der sozialen und ökonomischen Erscheinungen ringen muss, kann sich deswegen nur der reflektierenden Urteilskraft bedienen, weil sie über keine alllgemein gültigen Regeln verfügt. Wesentlich ist dabei, dass die Utreilskraft nicht über die moralischen Angelegenheiten entscheidet, weil die Moral ein Bereich ist, in dem nur die Vernunft entscheiden kann. Die Urteilskraft ermöglicht dagegen den Übegrang von moralischen Angelegenheiten zu den Problemen, die sich mit dem alltäglichen Leben und der Politik verbinden. Nur die reflektierende Urteilskraft kann also die einzelnen, immer konkreten und besonderen Fälle beurteilen.

Das politische, reflektierende Urteil ist für Kant eine Prozedur zur Feststellung intersubjektiver Übereinstimmung im öffentlichen Bereich. Diese „Prozedur" ist der Prozess, imaginativ von Standpunkten der anderen Menschen aus zu denken, und das auszubilden, was Kant als „erweiterte Denkungsart” bezeichnet hat: „Urteilskraft überhaupt ist das Vermögen, das Besondere als enthalten unter dem Allgemeinen zu denken. Ist das Allgemeine (die Regel, das Prinzip, das Gesetz) gegeben, so ist die Urteilskraft, welche das Besondere darunter subsumiert, [...] bestimmend. Ist aber nur das Besondere gegeben, wozu sie das Allgemeine finden soll, so ist die Urteilskraft bloß reflektierend. einen Mann von erweiterter Denkungsart anzeigt, wenn er sich über die subjektiven Privatbedingungen des Urteils, wo zwischen so viele andere wie eingeklammert sind, wegsetzen, und aus einem allgemeinen Standpunkte (den er dadurch nur bestimmen kann, daß er sich in den Standpunkt anderer versetzt) über sein eigenes Urteil reflektiert."22

Vermittelst der erweiterten Denkungart erheben sich damit die enthusiastisch urteilende Subjekte über ihre partikularen Interessen und gewinnen einen allgemeinen Standpunkt - ihre Einbildungskraft wird dann zu einer Bühne, auf der sie angesichts ganzer ,aufgeklärter” Menschheit auftreten. Wenn also die distanzierten Beobachter der Französichen Revolution sich in den Standpunkt des unparteiischen Zuschauer versetzen, können sie das Ganze der komplexen Zusammenhänge ergreifen und den allgemeinen Sinn der Ereignisse erkennen. Dieser Einblick eröffnet den Raum einer politischen Freiheit und lässt - nach Kant - die Revolution als ein epochales, lobenswertes Ereignis anerkennen, das der Menschheit Richtung auf die Zukunft weist.

${ }^{22}$ I. Kant, Kritik der Urteilskraft, (AA, 05: 295). 


\section{Schlussbemerkungen}

Abschließend möchte ich die wichtigsten Resultaten der vorglegten Ausführungen kurz und bündig betonen. Unsere Untersuchungen haben gezeigt: erstens, dass Kants ambivalente Haltung gegenüber der Revolution sich anhand Enthusiasmus-begriffs erklären läßt; zweitens, dass der politische Enthusiasmus immer auf die Ideen der Vernunft verweist und deswegen kein sinnliches Gefühl, sondern ein reiner, vernünftiger Affekt ist; drittens, dass die revolutionäre Handlung einen politischen Charakter nur gewinnt, wenn ein enthusiastisches Urteil der Zuschauer auf der Bühne der Öffentlichkeit „auftritt”; viertens, dass die Zuschauer ein aufgeklärtes Publikum bilden, das aus der Distanz die Revolution als "Geschichtszeichen” wahrnimmt und ihren tieferen Sinn fasst; fünftens, dass der Enthusiasmus struturanalog zum Erhabenen ist; sechstens, dass das enthusiastische Urteil in einer öffentlichen Debatte verifiziert werden muss und nur dann eine künftige Politik beeinflussen kann.

Was für einen Nutzen können wir aus diesen theoretischen Ergebnissen in den heutigen Debatten ziehen? Geben sie uns irgendwelchen, fruchtbaren Hinweis, wie man heutzutage die französischen Revolution verstehen und bewerten soll?

Im ersten Abschnitt dieses Artikels wurden die wichtigsten zeitgenössischen Interpretationslinien dieses „suspekten” Erbes genannt. Die Konservativen (und viele Neoliberalen) lehnen es im Alllgemeinen ab, Marxisten idealisieren es, die Übrigen vereinigen in ihrer Haltung beide widersprüchlichen Bewertungen - kurzum, sie behaupten, dass 1789 lobenswert sei, aber 1793 und die danach folgende Terror-Diktatur verpönt werden müsse. Kants Position scheint der dritten Option nah zu sein. Dieser Eindruck ist aber irreführend. Königsberger Philosoph lebte in einer feudalen Welt und die revolutionären Ereignisse musste er als eine Art von auratischen Erscheinungen, die zwar physisch ziemlich nah waren, aber geistig der fernen Galaxie angehörten, wahrnehmen. Das war für ihn ein Schimmer der neuen, idealen Ordnung, deswegen konnte er sich der Haltung eines unbeteiligten, neutralen Beobachters leisten. Der echte Sinn der Französischen Revolution steckte seiner Meinung nach in einem enthusiastisch reagierenden und denkenden Subjekt und nicht in den konkreten Ereignissen. Er verstand die Revolution als Geschichtszeichen, und ebendadurch als ein besonderes Gedankenexperiment, das das aufgeklärte Publikum in Betracht ziehen sollte. Wir befinden 
sich aber nicht in einer so komfortablen Lage, natürlich mit Ausnahme von denen, die den Wert und den Einfluß der Revolution diskreditiern. Wenn wir zumindest teilweise dieses Erbe und seine Bedeutung für die Gegenwart anerkennen, es als ein Gründungsereignis der modernen Welt bejahen, müssen wir uns zugleich damit abfinden, dass wir im gewissen Sinne die Komplizen der Revolution und zumindest die Nutznießer sowohl ihrer positiven Leistungen, als auch ihrer Gewalttätigkeit sind. Die Revolution gab uns die Menschen- und Bürgerrechte, die Wahlrechte, die immer noch gültigen Ideen der politischen Freiheit, Gleichheit, Menschenwürde etc. Sie versuchte dies alles in die Praxis umzusetzen und nicht nur theoretisch zu deklarieren. Andererseits zeigte die revolutionäre Handlung ihr schreckliches und gespenstisches Antlitz. Würde das eine simple und peinliche Formel: „die Geburt des Guten aus dem Geiste des Bösen” bedeuten? Nicht unbedingt. Nach der am weitesten verbreiteten Ansicht soll man in diesem Fall die Spreu vom Weizen trennen, d.h. demütig jene Schattenseite der Geburtsstunde unserer Demokratie akzeptieren und sie im Gedächtnis als die Mahnung für die Zukunft bewahren. Auf den ersten Blick scheint diese Antwort eine Stimme des gesunden Menschenverstandes zu sein, aber sie führt im Grunde genommen zur getarnten Ästhetisierung unseres Denkens über die französischen Revolution.

Wenn wir die Schreckensherrschaft der Jakobiner als das kontingente Abrutschen in die Barberei betrachten, dann abstrahieren wir im gewissen Sinne von dem politischen Gehalt der Revolution und sie zum Spektakel degradieren, das sich in einer anderen, fernen Welt abgespielt hat. Aus sicherer Entfernung lassen wir dieses Spektakel auf uns wirken und ziehen wir daraus ästhetisches Vergnügen (die massenhafte Produktion von literarischen Werken, Filmen, Dramas, TV Sendungen etc.). Der Mischmasch der Akzeptanz und der Verpönung weckt bei uns eine besondere Lust am Schrecklichem: ebenso wie Lukrez das ästhetische Vergnügen der Schaulustigen beim Schiffbruch durch die physische Sicherheit der Zuschauer schildert und begründet, so geniessen wir als Zuschauer das lange her vergangene Drama der Revolution aus sicherer Distanz. ${ }^{23}$ Sein ambivalenter, politischer Gehalt wird zum Objekt der ästhetischen Kontemplation oder leeren Unterhaltung. Auch die meisten Historiker, die eine solche ambivalente Haltung einnehmen, neutralisieren die finsteren Aspekte der französischen Revolution als Fehler, die niemals geschehen sollten. Problematisch ist in dieser Auffassung, dass alle uns bekannten Revolutionen immer mit dem schrecklichen Blutbad, mit dem Tod

${ }^{23}$ T. L. Carus, O naturze wszechrzeczy, tłum. E. Szymański, Warszawa 1957. 
von tausenden Menschen verbunden waren. Deswegen müssen wir uns vor allem in die Frage vertiefen, ob unsere wichtigsten postrevolutionären Ideen sich einfach von der "Gründungsgewalt” trennen lassen. Die Antwort, dass diese Ideen ihre eigene, lange und rein theoretische Geschichte haben, die man keinesfalls mit der Gewalt verbinden darf, ist eine faule Ausrede, weil es hier nicht um den philosophischen Diskurs, sondern um die konkrete, bestehende Wirklichkeit geht. Besonders heutzutage, wenn wir gegen die Globalisierung, gegen das Desaster der Finanzinstitutionen, die Migrantenkrise, den sogenannten „Konflikt zwischen Zivilisationen“ und besonders gegen die weltweit gewalttätigen Auseinandersetzungen zwischen Muslimen und Nichtmuslimen, ringen müssen, erhebt sich immer wieder die Frage nach der Legitimation unserer demokratischen Kultur. Das Erbe der Französischen Revolution gehört zu den Fundamenten unseres politischen Selbstbewußtseins, was die Notwendigkeit bedeutet, eine peinliche Vermutung zu überdenken, dass unsere Demokratie vielleicht keine „unschuldige”, vernünftige Einrichtung ist. Die Geschichte liefert uns die Lehre, dass die neue Ordnung sich auf dem Weg der Reformen, der graduellen Veränderungen nicht einführen lässt. Kant ahnte dieses Dilemma voraus, aber es war für ihn nur ein rein theoretisches Problem, das ihn zur Paradoxie geführt hat. Wir können uns nicht mehr mit der Feststellung der Pradoxie begnügen. Der symbolische Konflikt zwischen den Daten 1789 und 1793 wirft das Licht auf die gegenwärtigen Konflikte und vielleicht zwingt uns zum Umdenken unserer politischen und ethischen Prioritäten. Das bedeutet aber nicht, dass wir jetzt die Waffen gegen die Feinde ergreifen sollten, sondern, dass wir die Weichen auf die anderen Bahnen umstellen und nach den neuen Grundsätzen suchen müssen. In welche Richtung soll diese Suche verlaufen? Zur Beantwortung der Frage brauchen wir vieleicht jemanden, der wie Kant vor über zwei Jahrhunderten imstande wäre, einen tieferen Sinn in den revolutionären und scheinbar moralisch fragwürdigen Ereignissen in unserer Welt zu erblicken. 


\section{Literatur}

Arendt, H. Über die Revolution, München 1974.

Baszkiewicz, J., Meller, S., Rewolucja francuska 1789-1794. Społeczeństwo obywatelskie, Warszawa 1983.

Brzozowski, S., Eseje i studia o literaturze, t. 1, Wrocław 1990.

Dietzsch, S. Immanuel Kant. Biografia, tłum. K. Krzemieniowa, Warszawa 2005.

Furet, F., Interpreting the French Revolution, Cambridge 1978.

Kant, I. Gesammelte Schriften, Hrsg.: Bd. 1-22 Preussische Akademie der Wissenschaften, Bd. 23 Deutsche Akademie der Wissenschaften zu Berlin, ab Bd. 24 Akademie der Wissenschaften zu Göttingen. Berlin 1900ff.:

- Die Metaphysik der Sitten (AA 06)

- Der Streit der Fakultäten (AA 07)

- Beantwortung der Frage: Was ist Aufklärung? (AA 08)

- Kritik der Urteilskraft (AA 05)

Przybyszewska, S., Sprawa Dantona, Kraków 2003.

Vorländer, K., Kants Stellung zur französischen Revolution, in: Philosophische Abhandlungen. Hermann Cohen zum 70sten Geburtstag dargebracht, Berlin 1912, S. 247-269.

Wahnich, S., In defence of terror. Liberty or death in French Revolution, tr. D. Fernbach, London 2012.

Titus Lucretius Carus, O naturze wszechrzeczy, tłum. E. Szymański, Warszawa 1957. Żelazny, M., Idea wolności w filozofii Kanta, Torun 2001.

\section{Summary}

This article is an attempt to explicate the notion of political enthusiasm through which Immanuel Kant expressed his attitude to the French Revolution and to show the consequences of his interpretation for contemporary debates on the heritage of this founding event. The first section contains a brief reconstruction of major interpretative lines, which describe today the importance of the French Revolution for contemporary democracy. The next sections $(2,3,4,5,6)$ clarify the ambiguous attitude of Kant to the French Revolution, which from the moral perspective was unequivocally condemned by him, and from the aesthetic and political perspective - evaluated very favorably. The main concept is here a category of enthusiasm. Enthusiasm as a purely rational affect, as a collectively experienced, irrational euphoria, functions in the Kant's deliberations an important heuristic function, because it allows him to justify 
the positive meaning of the revolution, which at the level of empirical facts turned out to be an escalation of violence. The key to understanding this ambivalence is the category of publicity (Publizität) and the specific understanding of the concept of politics. The last section will show the consequences that stem from his position for contemporary debates on the legitimation crisis of liberal democracy.

Keywords: The French Revolution, politics, enthusiasm, public sphere, judgment, Immanuel Kant, Sanisława Przybyszewska, the legtimations crisis of the liberal democracy 